\title{
A Study on the Diagnostic Abilities of Ultrasound Scans in Assessing the Uterine Fibroids Against Magnetic Resonance Imaging Findings in the Same Subject Population
}

\section{Bedoor Al Omran ( $\sim$ drbedooralomran@gmail.com )}

Radiology Department, Bahrain Defence Force Hospital

Amal Mohamed Mehad

Royal College of Surgeons in Ireland - Bahrain

Simbarashe Matoi

Royal College of Surgeons in Ireland - Bahrain

Seemal Maqsood Abdul Qadir

Royal College of Surgeons in Ireland - Bahrain

Ayomide Peluola

Royal College of Surgeons in Ireland - Bahrain

Robin O'Sullivan

Royal College of Surgeons in Ireland - Bahrain

Nawal Dayoub

Obstetric and Gynecology Department, Assisted Reproduction and Gynecology Center London

\section{Research Article}

Keywords: Fibroid size, Ultrasound, Magnetic resonance imaging, Fibroid type, Fibroid site

Posted Date: September 29th, 2021

DOl: https://doi.org/10.21203/rs.3.rs-926951/v1

License: (c) (1) This work is licensed under a Creative Commons Attribution 4.0 International License. Read Full License 


\section{Abstract}

Background: This study was designed to evaluate the matching percentage among findings of the ultrasound scans to the magnetic resonance imaging in women with fibroids in Bahrain.

Methods: This descriptive, retrospective study was conducted from January 2016 to December 2018 including all the female patients referred from the Gynaecological Department to the Radiological Department in the Bahrain Defense Force Hospital for magnetic resonance imaging and ultrasound scan for fibroid evaluation. The data collected included their age, parity and nationality. The StatsDirect software was used to analyze the fibroids according to the site, size, type and number.

Results: The average age of the recruited 205 female patients for the study was 43 years, with $81.5 \%$ as Bahraini citizens and a mean parity of 2.3. Ultrasound scan findings matched the Magnetic Resonance Imaging in the posterior fibroid sites in $52.5 \%$ of cases, followed by anterior (38.1\%) and fundal (21.8\%). For fibroid size, Ultrasound scans matched Magnetic Resonance Imaging findings in $83.3 \%$ for fibroids between $>5$ to $\leq 10 \mathrm{~cm}$, in $78.3 \%$ for fibroids between $>2$ to $\leq 5 \mathrm{~cm}$, and in $36.8 \%$ for fibroids sized $\leq 2$ $\mathrm{cm}$. Fibroids more than $10 \mathrm{~cm}$ in size were in agreement for $33.33 \%$ of fibroids. Submucosal fibroids matched in just $29.4 \%$ of cases, but for the subserosal fibroids, it was $44.8 \%$. The matching score for more than 4 fibroids was $61.8 \%$, followed by single fibroids (54.8\%) and lastly for two fibroids $(34.8 \%)$.

Conclusions: Fibroid site and size had the highest matching rates amongst all the categories.

\section{Background}

Uterine fibroids (or uterine leiomyomas) are the main gynecological tumors occurring in up to $50 \%$ of the women of reproductive age globally [1]. More than $30 \%$ of the women over the age of 30 years are affected by the fibroids, and it is estimated that more than $70 \%$ of the women will be affected in their lifetime [2]. Since, most affected women do not encounter any distressing effects or symptoms, uterine fibroids often go undiagnosed [3]. However, fibroids do have an approximately 0.1 to $0.8 \%$ risk of transforming into malignant sarcomas. ${ }^{1}$ Affected women have an increased morbidity and an increased risk of encountering reproductive problems such as infertility and miscarriages [4].

Traditionally, the diagnosis of leiomyomas has been carried out using diagnostic imaging modalities, with ultrasound scans (USS). It has shown relatively high sensitivity and specificity, usually being the first-line imaging examination [5]. USS is used as the standard confirmatory modality because they are relatively cheap, accessible, harmless and can differentiate uterine fibroids from a pregnant' uterus or other adnexal tumors [6]. The two main routes used for ultrasonography are the transvaginal ultrasound scans (TVUSS) and transabdominal ultrasound scans (TAUSS) routes, and ideally both should be performed to detect, characterize and map the uterine fibroids [7]. TVUSS has a higher sensitivity for smaller fibroids and is capable of visualizing fibroids as small as $5 \mathrm{~mm}[7,8]$. However, when the uterus is retroverted or bulky, TAUSS is preferred because the uterine fundus could possibly be out of view transvaginally [7]. TAUSS has a wider field of view, increased transducer movement, more signal 
penetration ability, and offers the ability to examine other organs [9]. TAUSS itself has a limitation; it is difficult to be used in very obese patients and is less effective when the uterus is greater than $300 \mathrm{ml}$ in total volume [8]. Noticeably, USS has several disadvantages; it has a low efficacy, when there are multiple fibroids present, since these fibroids may then produce acoustic shadows through which sound fails to propagate [8]. It may miss smaller fibroids and be unable to determine the exact number and their location. USS may miss subserosal fibroids $[7,10]$. Another disadvantage associated with USS is that it also has considerable inter-observer/intra-observer variability or operator dependence, therefore, in the hands of a less skilled user, fibroids may be missed [7]. Overall, it could be interpreted that USS results have lesser reproducibility when compared to magnetic resonance imaging (MRI), which is the other diagnostic test for uterine fibroids [7].

Although MRI is more expensive,it is the most sensitive and accurate modality as it could diagnose, measure, localize and quantify all the lesions including very small fibroids [11-13]. Other than the higher sensitivity in identifying fibroids than USS, MRI alsodoes not use any ionizing radiation. MRI provides greater spatial resolution, greater contrast resolution, and superior multiplanar capabilities. Combined, this makes MRI a superior evaluator of the uterine zone anatomy (clearly differentiating subserosal, intramural and submucosal fibroids) and also superior in its localization of small and unusually located uterine fibroids and cervical fibroids $[14,15]$. Additionally, MRI can also diagnose other pelvic abnormalities and pathologies missed on the USS and is useful in assessing fibroid response to the treatment $[7,16]$.

Since physicians have a high probability of encountering women with uterine fibroids, and nearly $40 \%$ of them may have significant morbidity, knowledge of the diagnostic modalities for leiomyomas among them is essential for fibroid management. The aim of thisstudy is to investigate whether USS was as accurate as MRI in diagnosing fibroids in the female patients. This is the first descriptive, retrospective study conducted in the Kingdom of Bahrain evaluating the performances of MRI and pelvic USSs in diagnosing the uterine fibroids.

\section{Methods}

\section{Study design and sample selection}

This study was carried out recruiting all the adult female patients who underwent MRI and USS diagnostic tests for the diagnosis of fibroids at Bahrain Defense Force (BDF) Royal Medical Services Military Hospital during the period of January 2016 to December 2018. Patients were referred from the Gynecological Department to the Radiological Department, concerning the diagnosis and evaluation of fibroids, regardless of their age, parity, or nationality during this period. Therefore, this descriptive, retrospective study included both, the patients entitled for free care and private care patients. Patients with missing data or patients who underwent only one type of diagnostic modality were excluded.

\section{Analysis of Data}


The software used to analyze the data was StatsDirect statistical package version 3.1.22 (Cambridge, UK, 2018). Using this software, the patientswere analyzed according to their demographics such as age and nationality, parity, entitlement, symptoms, type of imaging conducted, as well as fibroid characteristics observed. Information on the clinical features of the patients observed was categorized into bleeding, irregular periods, pain, mixed symptoms, and asymptomatic. We also included the percentage of patients who underwent post procedureimaging along with patients who did not and the patients with unknown outcome of the care. The percentage of patients not identified with specific sub-categories of fibroid site, type, and number were also reported. Continuous variables have been presented as mean \pm SD and median with range. Categorical variables have been presented as percentage.

\section{Data extraction and diagnostic equipment}

All the official radiological reports of MRI and USS were reviewed. The USS was done at both the departments viz. Gynecological Clinics and radiological X-ray departments. However, some USSs in the Gynecological department were retained from the primary hand-written notes. In regard to the diagnostic equipment used, the USSs were performed using two different machines: Hitachi and General Electric (GE). USSs were carried out both trans-abdominally and trans-vaginally. Further, for MRI also, departmenthas two machines with different magnetic powers: GE was 3 teslas and Siemens with 1.5 teslas. Any patient with missing information was excluded from the study.

\section{Results}

\section{Patient characteristics}

In this study, a total of 205 female patients were diagnosed with fibroids after undergoing MRI and USS of the pelvis over the period of two years (from January, 2016 to December, 2018). The average age of the patients was 43 years. Among all the recruited patients, nearly $81.5 \%$ were Bahraini women and only $18.5 \%$ consisted of females from other nationalities. The majority of the women were entitled to free care $(73.7 \%)$ and the other $26.3 \%$ diagnosed received treatment under private care. The predominant clinical features observed were bleeding, irregular periods, and pain, with bleeding (23.9\%) being the most common solitary symptom across the patients. Moreover, the highest percentage of the females experienced mixed symptoms (32.7\%). 41 patients were found to be asymptomatic and $80 \%$ of the patients were with symptoms. Further, 105 patients (51.2\%) did not undergo procedure for the removal of fibroids, 91 patients $(44.4 \%)$ did, and the procedure history of a few patients $(4.4 \%)$ was unknown (Table 1). 
Table 1

History, clinical presentation, and characteristics of female patients with fibroids, admitted to BDF Hospital, Bahrain.

\begin{tabular}{|ll|}
\hline Age (years) & Mean \pm SD 42.6 \pm 10.1 /median (range) (20-78) \\
\hline Nationality & \\
Bahraini & $167 / 205(81.5 \%)$ \\
Others & $38 / 205(18.5 \%)$ \\
\hline Parity & Mean \pm SD $2.3 \pm 2.5 /$ median (range) $(0-15)$ \\
\hline Entitlement & \\
Private & $54 / 205(26.3 \%)$ \\
Entitle to free care & $151 / 205(73.7 \%)$ \\
\hline Symptoms & \\
Bleeding & $49 / 205(23.9 \%)$ \\
Irregular periods & $12 / 205(5.8 \%)$ \\
Pain & $36 / 205(17.6 \%)$ \\
Mixed & $67 / 205(32.7 \%)$ \\
asymptomatic & $41 / 205(20 \%)$ \\
\hline Procedure performed & \\
Yes & $91 / 205(44.4 \%)$ \\
No & $105 / 205(51.2 \%)$ \\
Unknown & $9 / 205(4.4 \%)$ \\
\hline
\end{tabular}

\section{Fibroid characteristics by MRI}

Most of the cases identified in the study presented with fundal fibroid location (38\%), followed by anterior (20.5\%) and posterior (19.5\%) sites. The least being the cervical fibroid site which was only found in $5.4 \%$ of patients.

Fibroid size is an important characteristic observed in the terms of diagnosis. The most common size of fibroids diagnosed by MRI was $>2$ to $\leq 5 \mathrm{~cm}$ ( $44.9 \%$ patients). Fibroids $>5$ to $\leq 10 \mathrm{~cm}$ in size were the second most common subcategory diagnosed (32.2\% of patients). Fibroids $\leq 2 \mathrm{~cm}$ and $>10 \mathrm{~cm}$ in size were seen in the smallest percentage of patients (Table 2). The location of the fibroid is also an important diagnostic characteristic.Intramural (38\%) was the most common fibroid type diagnosed by MRI in our patients, followed by subserosal $(28.3 \%)$. Very few cases were identified for the submucosal $(8.3 \%)$ and mixed (14.6\%) fibroid types. 
Table 2

Fibroid characteristics observed under Magnetic Resonance Imaging in patient sample.

\begin{tabular}{|ll|}
\hline Uterine volume cm3 & Mean \pm SD 398 \pm 376 median (range) (25-2479) \\
\hline Fibroid site & \\
Anterior & $42 / 205(20.5 \%)$ \\
Posterior & $40 / 205(19.5 \%)$ \\
Lateral & $21 / 205(10.2 \%)$ \\
Fundal & $78 / 205(38 \%)$ \\
Cervical & $11 / 205(5.4 \%)$ \\
Complex & $12 / 205(5.9 \%)$ \\
Not identified & $1 / 205(0.5 \%)$ \\
\hline Fibroid size & \\
0 to $\leq 2$ cm & $38 / 205(18.5 \%)$ \\
$>2$ cm to $\leq 5 \mathrm{~cm}$ & $92 / 205(44.9 \%)$ \\
$>5$ cm to $\leq 10 \mathrm{~cm}$ & $66 / 205(32.2 \%)$ \\
$>10$ cm & $9 / 205(4.4 \%)$ \\
\hline Fibroid type & \\
Sub serosal & $58 / 205(28.3 \%)$ \\
Submucosal & $17 / 205(8.3 \%)$ \\
Intramural & $78 / 205(38 \%)$ \\
Mixed & $30 / 205(14.6 \%)$ \\
Not identified & $22 / 205(10.7 \%)$ \\
\hline Fibroid number & \\
\hline fibroid & $73(35.6 \%)$ \\
\hline fibroids & $23(11.2 \%)$ \\
Multiple fibroids & $5(2.4 \%)$ \\
\hline
\end{tabular}

Further, approximately half of the patients diagnosed by MRI showed multiple fibroids $(>3)$ [102 patients $(49.8 \%)]$. The second most common type was single fibroids (35.6\%) and these were followed by two 
fibroids (11.2\%). The fibroid numbers in a small percentage of patients were not identified in this study.

\section{USS findings weighed against MRI findings}

In regard tothe fibroid site, the highest percentage of the matched scan to the MRI were in the posterior fibroid sites $(52.5 \%)$, showing that more than half of the scan cases matched the gold standard diagnostic method. Anterior fibroid sites followed at $38.1 \%$ and the fundal at $21.8 \%$. Cervical fibroid sites had $18.2 \%$ of the scans matching to the MRI and the match rates were not good for the lateral fibroid sites $(9.5 \%)$ (Table 3$)$. 
Table 3

Ultrasound scan of patients with fibroids correlating with MRI findings.

\section{MRI findings USS matching MRI findings}

$\begin{array}{lll}\text { Fibroid site } & & \\ \text { Anterior } & 42 / 205(20.5 \%) & 16 / 42(38.1 \%) \\ \text { Posterior } & 40 / 205(19.5 \%) & 21 / 40(52.5 \%) \\ \text { Lateral } & 21 / 205(10.2 \%) & 2 / 21(9.5 \%) \\ \text { Fundal } & 78 / 205(38 \%) & 17 / 78(21.8 \%) \\ \text { Cervical } & 11 / 205(5.4 \%) & 2 / 11(18.2 \%) \\ \text { complex } & 12 / 205(5.9 \%) & 0 / 12(0 \%)\end{array}$

Fibroid size

0 to $\leq 2 \mathrm{~cm} \quad 38 / 205(18.5 \%) \quad 14 / 38(36.8 \%)$

$>2 \mathrm{~cm}$ to $\leq 5 \mathrm{~cm} \quad 92 / 205(44.9 \%) \quad 72 / 92(78.3 \%)$

$>5 \mathrm{~cm}$ to $\leq 10 \mathrm{~cm} \quad 66 / 205(32.2 \%) \quad 55 / 66(83.3 \%)$

$>10 \mathrm{~cm} \quad 9 / 205(4.4 \%) \quad 3 / 9(33.3 \%)$

Fibroid type

Sub serosal $\quad 58 / 205(28.3 \%) \quad 26 / 58(44.8 \%)$

Submucosal $\quad 17 / 205(8.3 \%) \quad 5 / 17(29.4 \%)$

Intramural $\quad$ 78/205(38\%) $\quad 35 / 78(44.9 \%)$

mixed $\quad 30 / 205(14.6 \%) \quad 3 / 30(10 \%)$

Fibroid number

1 fibroid $\quad 73 / 205(35.6 \%) \quad 40 / 73(54.8 \%)$

2 fibroids $\quad 23 / 205(11.2 \%) \quad 8 / 23(34.8 \%)$

3 fibroids $\quad 5 / 205(2.4 \%) \quad 2 / 5(40 \%)$

Multiple fibroids $\quad 102 / 205(49.8 \%) \quad 63 / 102(61.8 \%)$

A trend in the fibroid size was also noted revolving around the matching of US scans to the MRI findings. The highest percentages of matching findings were with fibroid sizes between $>5$ to $\leq 10 \mathrm{~cm}(83.3 \%)$, followed by $>2$ to $\leq 5 \mathrm{~cm}$ and 0 to $\leq 2 \mathrm{~cm}$, respectively. Surprisingly, fibroids more than $10 \mathrm{~cm}$ in size were observed to have the lowest match (33.3\%). Moreover, the highest matched scans for the fibroid types were observed in intramural (44.9\%) and subserosal (44.8\%) compared to the mixed types being only $10 \%$ (Table 3). 
The highest agreement found in identifying the fibroid number was in the presence of multiple fibroids at $61.8 \%$, followed by single fibroid at $54.8 \%$. The lowest matching was observed with two fibroids at $34.8 \%$ (Table 3).

\section{Discussion}

Previously published studies have compared the merits of USS and MRI findings with regards to the fibroid management. It has been cumulatively observed that MRI has more sensitivity and better specificity, indicating the numbers of fibroids present and their location more accurately [14]. Consequently, MRI has been widely considered to represent the gold standard in such radiological investigations. In one recent study, more than half the patients who underwent USS were found to have additional gynecological problems after undergoing MRI [16]. For these reasons, MRI is seen as the gold standard investigationas it can detect other gynecological abnormalities in addition tothe fibroids. It has been noted that women with fibroids can present with non-specific symptoms and on the contrary, they can also be asymptomatic $[1,17]$. In this study, $20 \%$ of the patients were found to be asymptomatic. Typical symptoms observed in the case of fibroids were infertility, dysmenorrhea, pelvic pain and menorrhagia, which are broad and non-specific $[3,18]$.

Inastudy by Dueholm and colleagues (2002), it was reported that the average number of fibroids located by MRI were $15 \pm 2.8$ compared to transvaginal USS which located $8 \pm 2.6$ fibroids. USS missed more fibroids than MRI and the discrepancy almost doubled when fibroids were classified according to both the position and uterine wall embedment [19]. Levens' study showed that MRI is twice as sensitive as USS in diagnosing the fibroids with a rate of $80 \%$ compared to $40 \%$ [20]. Our data showed that the best correlation between USS and MRI findings were in the cases with multiple fibroids over the three categories as shown in Table 3. This finding needs to be considered with caution, as we assessed the matching in the group of patients with over 3 fibroids without being specific of their number. It could be explained as when USS detected 4 fibroids and MRI confirmed the presence of more fibroids in the same patient, the data entry was still considered as matched. Noticeably, the observation which is accurate and clear is that when there is only one fibroid, the chance of matching with the scan was over $50 \%$.

Detection of subserous fibroids using USS showed to have a reduced sensitivity with some fibroids being undetectable or completely missed in some cases [21]. In our study, USS matched the MRI findings in $44.8 \%$ of the subserosal fibroids. Our study demonstrated variable matching abilities between USS and MRI in different fibroid types. The data of the present study further confirmed that submucosal fibroids are usually difficult to be differentiated on the USS scan. Only $29 \%$ of those cases matched the MRI findings. Other contributing factors would have added to the equation such as the size and the site. Contrary to our finding, a study by Ahmad group found USS to have a higher specificity and positive prediction value (PPV) than MRI in the diagnosis of the submucous fibroids.

In the study conducted by De La Cruz et al., fibroids were classified according to size, site, and number, but no percentages were given for the rate of occurrence for each individual characteristic of fibroids. The 
best matching site was the posteriorly located fibroid in the present study. This could be due to the angle of the vagina, where the posterior wall would be more accessible and nearer to the TVUSS. Surprisingly, our study showed that the cervical fibroids had alower matching rate than the MRI. This could simply be due to the mislabeling as anterior or posterior rather than cervical. Determining the accurate size of the fibroids is crucial forits management plan. In a previous study analyzing fibroid size, USS underestimated the size of a large $16.6 \mathrm{~cm}$ diameter fibroid to be $12.5 \mathrm{~cm}$ [22]. Findings of our study were in line with this previously published data and showed that fibroids over $10 \mathrm{~cm}$ correlatedpoorlywith the USS (matching only $33.3 \%$ of cases) (Table 3 ). However, the USS findings in our study matchedvery well with the MRI findings in case of the fibroids between 2 and $10 \mathrm{~cm}$ in diameter.

There are limitations to all of these studies, as technical skills play a crucial role in producing accurate USS results. The reality is that different levels of experience and competence influence the findings, as do the differences between the models of USS and MRI machines used. In addition, missing data reported as non-identified could also skew the results. Increasing the sample size of the study could help to reduce the error, but it will not entirely eliminate it.

\section{Conclusion}

Although MRI has been consideredsuperior in fibroids diagnosis based on its various properties, it does not negate the merits of USS as a preliminary diagnostic tool, especially where MRI is not readily accessible. In the present study, USS and MRI findings correlated reasonably well in terms of fibroid site and size.

\section{Abbreviations}

USS: ultrasound scans

TVUSS: transvaginal ultrasound scans

TAUSS: transabdominal ultrasound scans

MRI: magnetic resonance imaging

PPV: positive prediction value

\section{Declarations}

\section{Ethics approval and consent to participate}

The ethical approval was obtained from the research and research ethics committee at BDF hospital prior to conducting this research (reference number 338). An ethical approval form was also obtained from the RCSI Bahrain summer research to enroll the medical students (Amal Mohamed Mehad, Simbarashe 
Matoi, Seemal Maqsood, Ayomide) to participate in this paper. The research was conducted in accordance with the 1964 Helsinki Declaration.

\section{Consent for publication}

Not applicable

\section{Availability of data and materials}

Not applicable

\section{Competing interests}

The authors declare that they have no competing interests.

\section{Funding}

This research did not receive any specific grant from the funding agencies in the public, commercial, or not-for-profit sectors.

\section{Authors' contributions}

BAO and ND contributed to the study conception and design. The data collection was performed by SM. The first draft of the manuscript was written by AMM, SM and AP.

The final version was revised by BAO and AMM. The English proofing was done by RO. The project supervision was done by ND and all authors commented on previous versions of the manuscript. All authors read and approved the final manuscript. Authors testify that all persons designated as authors qualify for authorship and have checked the article for plagiarism.

\section{Acknowledgments}

We would like to thank the RCSI summer research school and BDF hospital for helping in conducting this research.

\section{References}

1. Woźniak A, Woźniak S. Ultrasonography of uterine leiomyomas. Przeglad menopauzalny= Menopause Rev. 2017;16:113.

2. Aboulghar M, Al-Inany H, Aboulghar M, Serour G, Mansour R. The effect of Intramural Fibroids on the Outcome of IVF. Middle East Fertil Soc J. 2004;9:263-267.

3. Bolan C, Caserta MP. MR imaging of atypical fibroids. Abdominal Radiol. 2016;41:2332-49.

4. Morin SJ, Schlaff WO. Optimal approaches to fibroid management: consider fibroid location and the patient's pregnancy plans. Contemporary OB/GYN. 2017;62:21-6. 
5. Wise LA, Radin RG, Palmer JR, Kumanyika SK, Boggs DA, Rosenberg L. Intake of fruit, vegetables, and carotenoids in relation to risk of uterine leiomyomata. Am J Clin Nutr. 2011;94:1620-31.

6. Stewart EA. Uterine fibroids. N Eng J Med. 2015;372:1646-55.

7. Khan A, Shehmar M, Gupta J. Uterine Fibroids: Current Perspectives. Int J Women's Health. 2014;6:95-114.

8. McLucas B. Diagnosis, Imaging and Anatomical Classification of Uterine Fibroids. Best Pract Res Clin Obstet Gynaecol. 2008;22:627-642.

9. Testa AC, Di Legge A, Bonatti M, Manfredi R, Scambia G. Imaging techniques for evaluation of uterine myomas. Best Pract Res Clin Obstet Gynaecol. 2016;34:37-53.

10. De La Cruz M, Buchanan E. Uterine Fibroids: Diagnosis and Treatment. Am Fam Physician. 2017;95:100-7.

11. Badawy M, Elkholi D, Sherif M, Hefedah M. Magnetic Resonance Imaging for Diagnosis of Pelvic Lesions associated with Female Infertility. Middle East Fertil Soc J. 2015;20:165-75.

12. Parker W. Etiology, Symptomatology, and Diagnosis of Uterine Myomas. Fertil Steril. 2007;87:72536.

13. Hossain MZ, Rahman MM, Ullah MM, Mukthadira M, Akter FA, Jahan AB, et al. A Comparative Study of Magnetic Resonance Imaging and Transabdominal Ultrasonography for the Diagnosis and Evaluation of Uterine Fibroids. Mymensingh Med J. 2017;26:821-7.

14. Rashid S, Chou Y, Tiu C. Ultrasonography of Uterine Leiomyomas. J Med Ultrasound. 2016;24:3-12.

15. Ahmad R, Sadek S, Ragheb A. Evaluation of the Uterine Cavity by Magnetic Resonance Imaging, Three Dimensional Hysterosonography and Diagnostic Hysteroscopy in Women with Pre- and Postmenopausal Bleeding. Middle East Fertil Soc J. 2015;20:70-8.

16. Vu K, Fast A, Shaffer R, Rosenberg J, Dababou S, Marrocchio C, et al. Evaluation of the Routine Use of Pelvic MRI in Women presenting with Symptomatic Uterine Fibroids: When is Pelvic MRI useful? J Magn Reson Imaging. 2019;49:e271-81.

17. Elkattan E, Kamel R, Elghazaly H, ElAriki E. Can Three-Dimensional (3D) Power Doppler and Uterine Artery Doppler differentiate between Fibroids and Adenomyomas? Middle East Fertil Soc J. 2016;21:46-51.

18. Abbas H, Awad I, Alharbi E, Alameri H, Althubaiti S, Ashkar L. Prevalence and Incidence of Uterine Fibroid at King Abdulaziz University Hospital Saudi Arabia. Clin Med Diagn. 2016;6:45-48.

19. Dueholm M, Lundorf E, Hansen E, Ledertoug S, Olesen F. Accuracy of Magnetic Resonance Imaging and Transvaginal Ultrasonography in the Diagnosis, Mapping, and Measurement of Uterine Myomas. Am J Obstet Gynecol. 2002;186:409-15.

20. Levens E, Wesley R, Premkumar A, Blocker W, Nieman L. Magnetic Resonance Imaging and Transvaginal Ultrasound for Determining Fibroid Burden: Implications for Research and Clinical Care. Am J Obstet Gynecol. 2009;200:537-e1. 
21. De La Cruz M, Buchanan E. Uterine Fibroids: Diagnosis and Treatment. Am Fam Physician. 2017;95:100-7.

22. Spielmann A, Keogh C, Forster B, Martin M, Machan L. Comparison of MRI and Sonography in the Preliminary Evaluation for Fibroid Embolization. Am J Roentgenol. 2006;187:1499-504. 